\title{
Study of the Electrophysical Properties of Composite Varistors Based on Zinc Oxide and Polymer (Polyaniline)
}

\author{
Sajedeh Mohammadi Aref, ${ }^{1}$ Hassan Bidadi, ${ }^{1}$ and Shamestan Hasanli ${ }^{2}$ \\ ${ }^{1}$ Department of Solid State \& Electronics, Faculty of Physics, University of Tabriz, Tabriz, Iran \\ ${ }^{2}$ Institute of Physics, Azerbaijan National Academy of Sciences, Baku, Azerbaijan \\ Correspondence should be addressed to Hassan Bidadi, bidadi@tabrizu.ac.ir
}

Received 22 November 2009; Revised 14 March 2010; Accepted 11 May 2010

Academic Editor: Robert Shanks

Copyright ( 2010 Sajedeh Mohammadi Aref et al. This is an open access article distributed under the Creative Commons Attribution License, which permits unrestricted use, distribution, and reproduction in any medium, provided the original work is properly cited.

\begin{abstract}
In this experimental work, current-voltage characteristics of composite varisrors prepared on the base of zinc oxide with different weight percentages of polyaniline and temperature dependence of the samples characteristics have been studied. The activation energy of donor levels as well as the existence of hysteresis loop has been investigated. The comparison of experimental results shows that by increasing the polymer percentage in the varistor structure as long as the nonlinear behavior is conserved, the threshold voltage increases. This comparison also indicates that increasing the polymer content in the varistor structure causes the temperature dependence of threshold voltage to decrease. It is also concluded that, by the increase of polymer content in the varistor structure, the activation energy of donor levels and the hysteresis value are increased.
\end{abstract}

\section{Introduction}

Nowadays, for protecting the electronic and microelectronic circuits, and electrical equipment from overvoltages, the nonlinear resistors based on composite materials are widely utilized. By conversion of microelectronics into nanoelectronics, the need for low-voltage limiters will grow even more [1]. Zinc oxide varistors are electronical polycrystalline ceramic materials whose electronical behavior is determined by grain boundaries. These materials have important technological applications in electronic circuits for preventing them from overvoltages [2]. Pure zinc oxide is a nonstoichiometric n-type semiconductor whose current-voltage behavior is linear and additives such as $\mathrm{Al}_{2} \mathrm{O}_{3}, \mathrm{CrO}$, and $\mathrm{Bi}_{2} \mathrm{O}_{3}$, are used to make it become nonlinear. Adding these materials causes a number of crystal imperfections to be formed in grains and also grain boundaries. These imperfections have the role of donors in grains and acceptors in grain boundaries [3].

As a result of imperfections due to covalent bonding, certain surface states in band gap are created that affect the electrical properties of varistors [4]. In a varistor, transition from a high resistance region (pre-breakdown region) to a lower resistance region (upturn region) is not sharp, but the relation between current and voltage is nonlinear which follows the form $I=k V^{\alpha}$, where $\alpha$ is called the coefficient of nonlinearity. The above relation shows that for a constant current, with increasing $\alpha$ value, the voltage changes in the transition region decreases. The higher the $\alpha$ value, the better the quality of varistor. The nonlinear coefficient $(\alpha)$ is very important for protecting the electrical equipment. The advantage of $\mathrm{ZnO}$ varistors over the $\mathrm{SiC}$ varistors comes from their higher $\alpha$ values $[3,5]$.

The common understanding of the nonlinear electricalthermal switching and device operation is based on the coupling of nonlinear electricity, Joule heating, and positive thermal resistance coefficient (PTRC) in the composites.

This allows us to combine the nonlinear electrical behavior needed with polymer processing properties to control a high field or electrostatic discharge protection for electronics.

Recently, new composite polymer-varistors for low voltage applications are being prepared. In comparison, new composite varistors have lower threshold voltages than those of the available composite varistors. In addition, their $\alpha$ values are also very high [6]. The greatest advantage of composites is their stability and hardness in addition to 

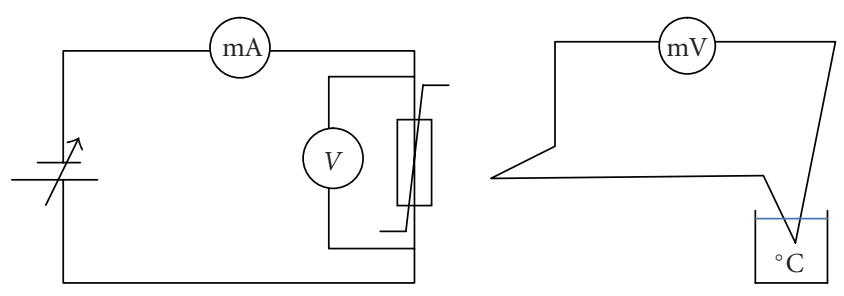

FIGURE 1: The schematic of varistor in the circuit and measurement of its temperature.

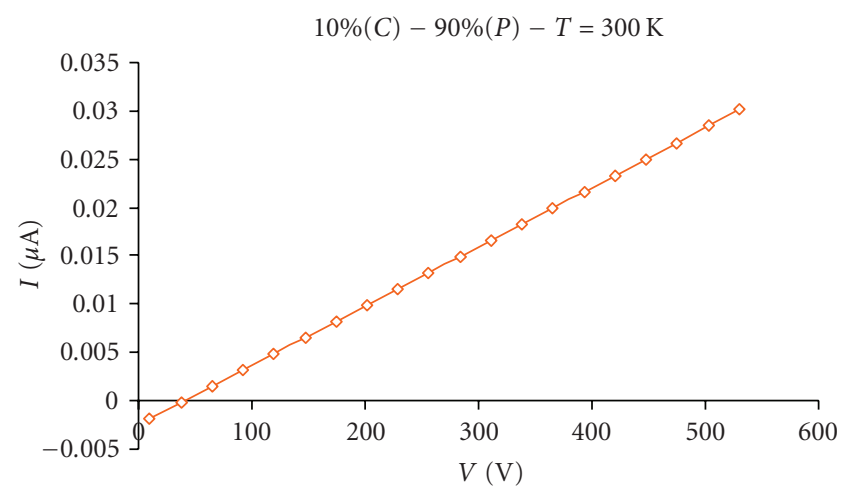

Figure 2: The $I-V$ characteristic for sample no.1 at room temperature.

their lightness. Composites have high mechanical resistance against heat and corrosion and are also flexible [7]. Since their discovery, polymers were regarded to be used solely as insulators; however, recently it is possible to produce conductive polymers with conductivity higher than that of copper. Usually structure complexity causes conductive polymers to act as semiconductors. The most notable difference between conductive polymers and inorganic semiconductors is the mobility, which until very recently was dramatically lower in conductive polymers than their inorganic counterparts. Their conductivity is close to Silike semiconductors. Polymers' electronic structure is similar to that of insulators or semiconductors that the valance band is completely full and the conduction band is empty. The energy gap between these two bands is usually bigger than $1 \mathrm{eV}$ and sometimes reaches several electron volts. The band theory is not true about polymers. Because of the covalence band between atoms, they form polymer chains having weaker mutual intermolecular effects. Polymer's electronic conductivity is caused by mobile carriers which appear in electronic system by doping process. Doping (positive or negative) induces electric charge in polymer chain which causes small change in "solitons", "polarons" or "bipolarons". These charge islands are responsible for electric conductivity in conductive polymers. "Solitons", "polarons" and "bipolarons" are bosons, and Pauli exclusion principle is not true for them. Macroscopic conductivity not only depends on electron movement through the chain but also depends on the electron transition between different chains. The increase of conductivity in conductive polymers depends on carriers' mobility [8].

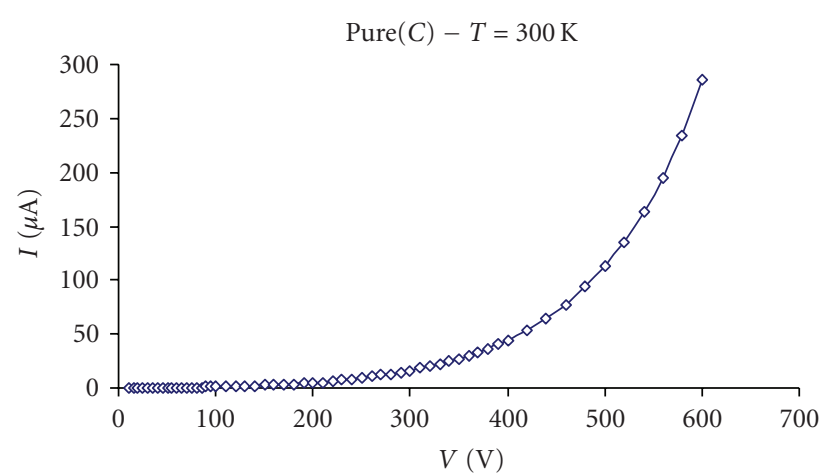

FIGURE 3: The $I-V$ characteristic for sample no. 5 at room temperature.

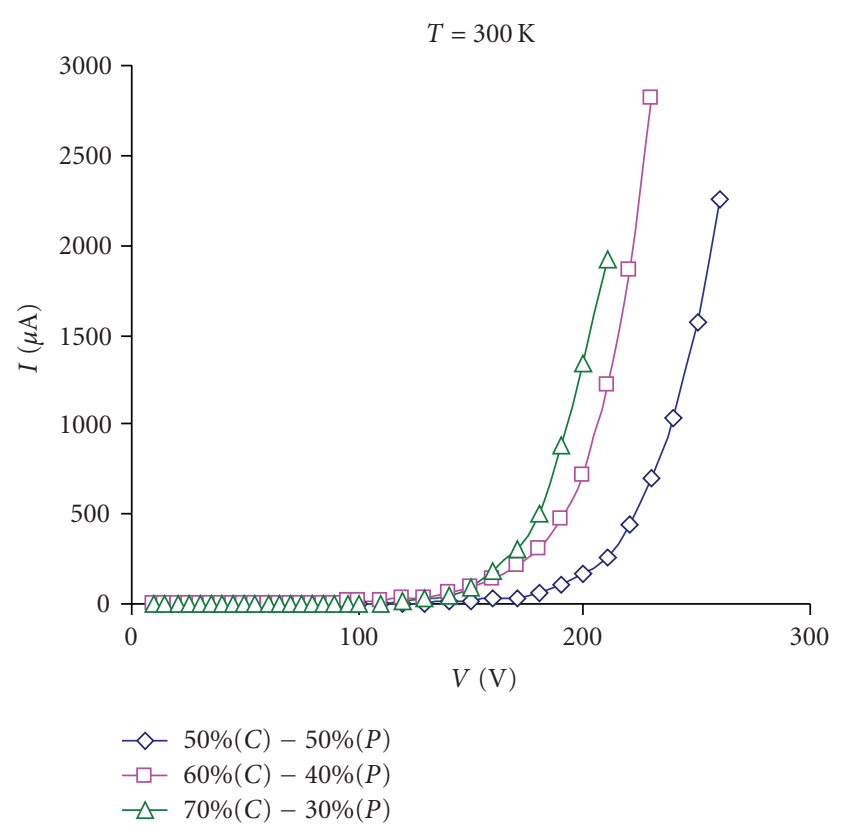

Figure 4: The $I-V$ characteristic for samples no.2, no.3, and no.4 at room temperature.

Based on resent studies, polymer matrix is considered more than just a binder having specific effects. Organic Polymeric Varistors can be used as low breakdown voltage varistors, to which increasing interest is caused by their application in electronic devices as chips and computer processors.

\section{Experimental Methods}

Synthesis of $\mathrm{ZnO}$-based varistors is accomplished according to the principles of ceramics. The ceramic matrix used for this aim is composed of $97 \% \mathrm{ZnO}$ plus $3 \%$ other oxide additives. In this case, the additives are $\mathrm{Sb}_{2} \mathrm{O}_{3}, \mathrm{Bi}_{2} \mathrm{O}_{3}, \mathrm{MnO}$, $\mathrm{CoO}, \mathrm{Cr}_{2} \mathrm{O}_{3}$, and $\mathrm{Ni}_{2} \mathrm{O}_{3}$. In the process of synthesis, the precursor and terminal materials are in solid forms, and the diffusion process takes place between the particles of the precursor materials. To increase the diffusion effect between the precursor particles, high temperatures are used in such 


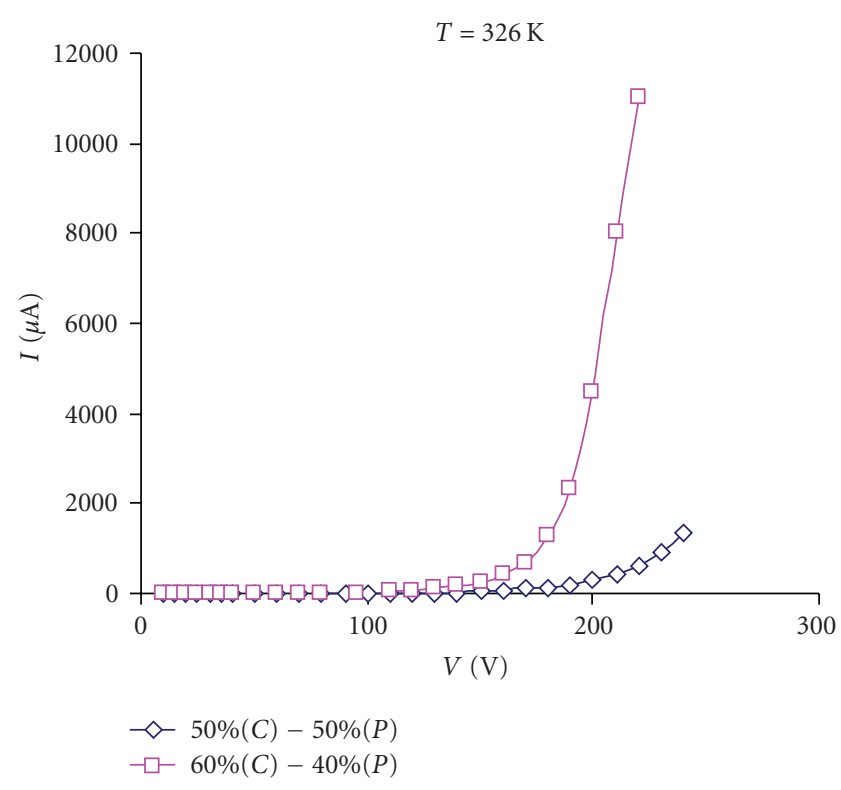

FIGURE 5: The comparison of $I-V$ characteristic for samples no.2 and no. 3 at a higher temperature.

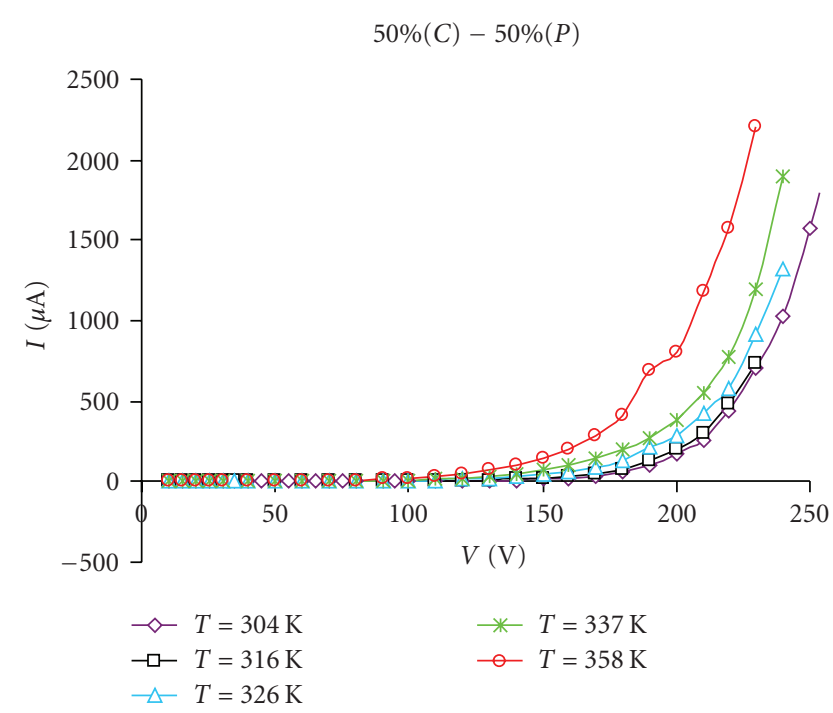

FIGURE 6: The variation of the $I-V$ characteristic with temperature for sample no.2.

a way that ceramic synthesis takes place at temperatures as high as $2300 \mathrm{~K}$. For the simplicity of diffusion, the particles are considered as small as possible. For making particles having the closest contacts in the process of synthesis, the mixture prepared from different materials is pressed under high pressures $\left(20 \mathrm{MPa}\right.$ at $\left.160^{\circ} \mathrm{C}\right)$.

These presently investigated composite varistors are prepared in National Academy of Sciences of Azarbayjan by preparation of required powders, mixing them according to the principles of ceramics, and pressing them at $160^{\circ} \mathrm{C}$. The composite varistors which are made of $\mathrm{ZnO}$ and different percentages of polyaniline are in the form of thin circular discs with $25 \mathrm{~mm}$ diameter and $180 \mu \mathrm{m}$ thickness. They are

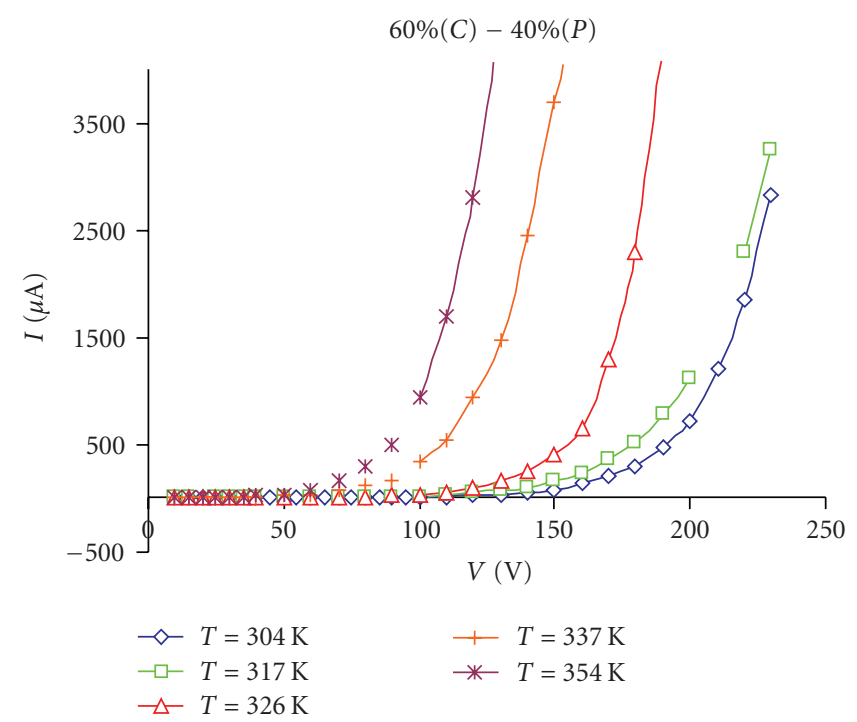

FIGURE 7: The variation of the $I-V$ characteristic with temperature for sample no.3.

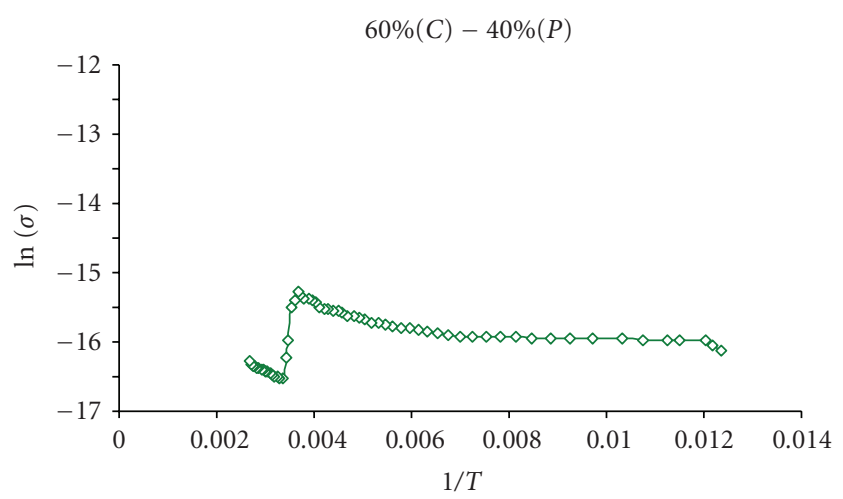

Figure 8: The $\ln \sigma \sim 1 / T$ curve for sample no.3.

precleaned by pure water and alcohol in an ultrasonic cleaner system and then dried by compressed air.

Both sides of the samples were coated by silver paste. It should be mentioned that in order to have good electric contact, the silver paste should completely get dried. Then the sample was located in a circuit and by applying different voltages the circulating current through the sample was measured. This measurement was taken at room temperature first, then by using a heater the temperature was varied and it was measured by an iron-constantan thermocouple. The current-voltage characteristic of all samples was measured at different temperatures.

In this experimental work, this process was repeated for 5 different composite varistors that had the following peculiarities:

(i) $10 \% \mathrm{ZnO}$ ceramic matrix, $90 \%$ polyaniline,

(ii) $50 \% \mathrm{ZnO}$ ceramic matrix, 50\% polyaniline,

(iii) $60 \% \mathrm{ZnO}$ ceramic matrix, 40\% polyaniline,

(iv) $70 \% \mathrm{ZnO}$ ceramic matrix, 30\% polyaniline, 


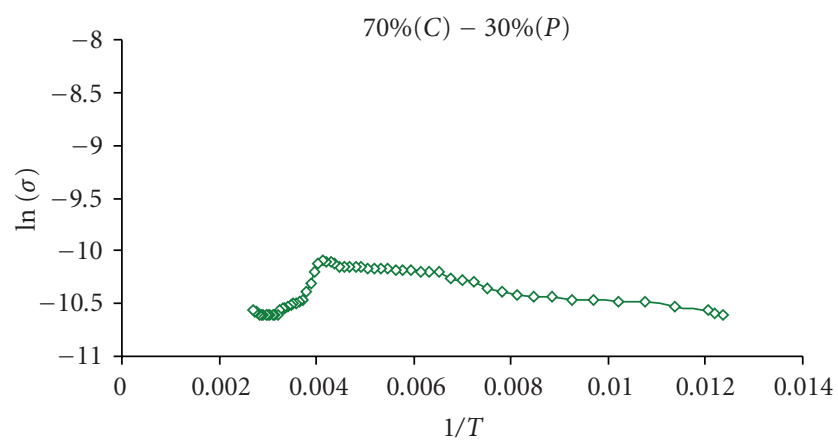

Figure 9: The $\ln \sigma \sim 1 / T$ curve for sample no.4.

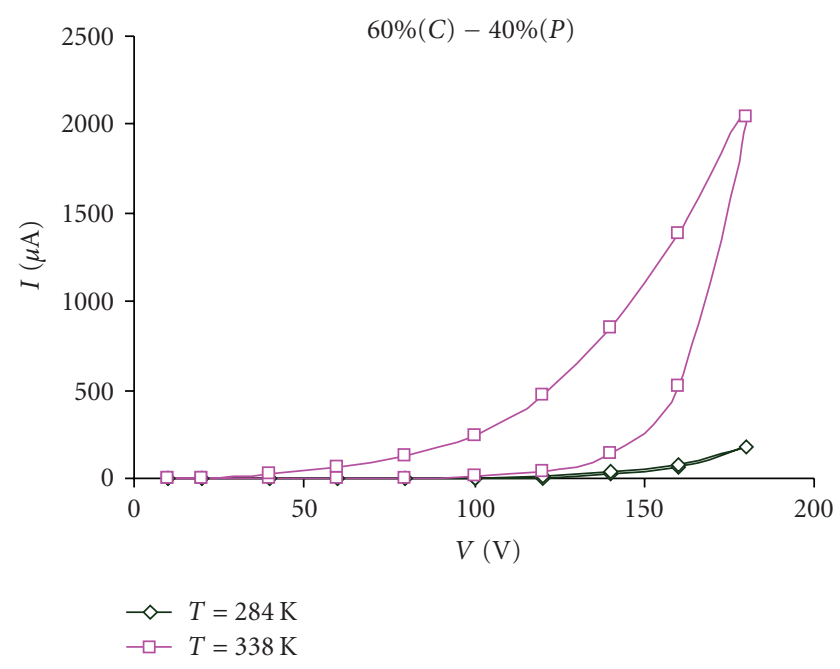

Figure 10: The hysteresis loop for sample no.3.

(v) $\mathrm{ZnO}$ based varistor with $0.00 \%$ polyaniline.

Finally the obtained results were compared with each other.

\section{Results and Discussion}

For studying the effect of polyaniline increment on $\mathrm{ZnO}$ varistor's threshold voltage, each of the samples was placed in the circuit shown in Figure 1 and its $I-V$ characteristics was studied at room temperature.

By plotting the $\ln \sigma \sim 1 / T$ curve and evaluating its slope, the activation energy of donor levels was calculated:

$$
\begin{gathered}
\sigma=\sigma_{o} \exp \left[\frac{-\left(E_{g}+\Delta E\right)}{2 k T}\right], \\
\operatorname{tg} \alpha_{1}=-\frac{\Delta E}{2 k} \quad \operatorname{tg} \alpha_{2}=-\frac{E_{g}}{2 k} .
\end{gathered}
$$

The hysteresis loop exhibited by the $I-V$ characteristic was simply plotted by increasing and then decreasing the applied voltage in the range of $0 \rightarrow 200 \mathrm{v} \rightarrow 0$. The $I-V$ characteristics for samples no.1-that is composed of $90 \%$ polyaniline and only $10 \% \mathrm{ZnO}$ ceramic matrix-had approximately linear

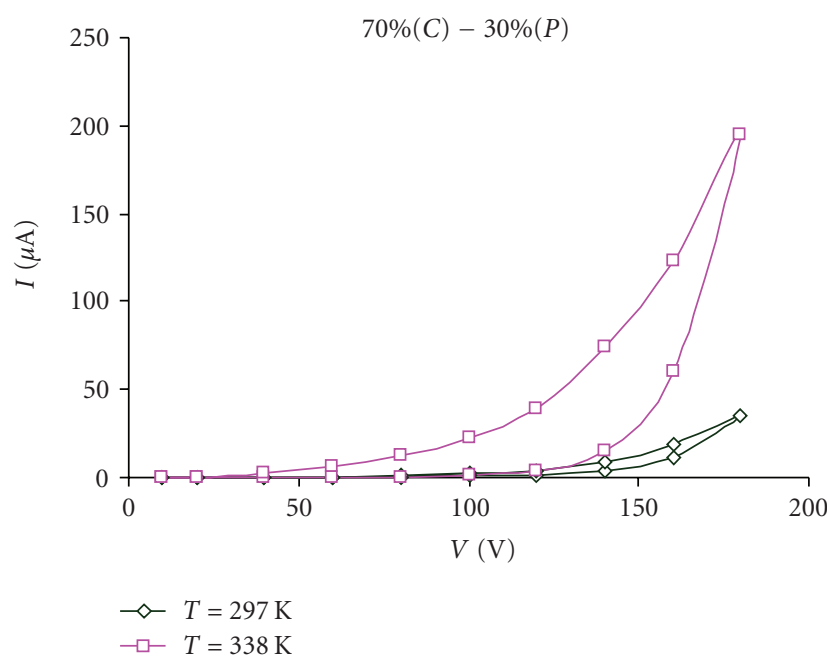

FIgure 11: The hysteresis loop for sample no.4.

behavior as shown in Figure 2. This shows that the nonlinearity of sample is induced by $\mathrm{ZnO}$ ceramic matrix. If the polymer content is much higher than that of $\mathrm{ZnO}$ ceramic matrix, the nonlinear behavior of resistor will vanish and current-voltage characteristics will be linear. So it is essential that the content of $\mathrm{ZnO}$ ceramic matrix and polyaniline must approximately be the same. For small applied electric fields, varistor acts as a dielectric, but by increasing the applied voltage, the resistance of varistor decreases suddenly and the varistor's conductance and its current are increased. A $\mathrm{ZnO}$-based varistor without polymer content which shows nonlinear characteristic is depicted in Figure 3. The comparison of $I-V$ characteristics of samples no.2, no. 3 and no. 4 with each other shows that by increasing the polyaniline percentage in the varistor structure, the threshold voltage is increased, (Figure 4). Adding polymer merely changes the value of threshold voltage.

The study of temperature dependence of $I-V$ characteristics of a varistor indicates that by increasing the varistor temperature, its threshold voltage is decreased. On the other hand, the temperature increase causes the resistance to decrease and threshold voltage becomes lower. It is also seen that the higher the polyaniline content in the varistor structure, the heavier the threshold voltage decrease by increasing the temperature, (Figure 5).

The temperature increase of a composite varistor may cause destruction in the varistor due to the polymer dissociation. The nonlinear coefficient for composite varistors is defined as

$$
\alpha=\frac{d(\ln I)}{d(\ln V)} \longrightarrow d(\ln I)=\alpha d(\ln V) .
$$

The value of $\alpha$ for samples no.2, no. 3 and no. 4 was evaluated to be $4.2,4.3$, and 4.4 , respectively. The $\alpha$ value for an ideal varistor is infinite. In the pre-breakdown zone, the $I$ $V$ characteristics are linear. In the upturn zone, it is linear, too. The difference between these two regions is that the 
slope of the $I-V$ curve is higher in the upturn zone. In other words, the varistor possesses lower resistance in this region. By increasing the temperature, the samples' resistance in both zones is decreased.

In nonlinear zone, the $I-V$ characteristics are not linear. In this region, the sample behaves as a non-Ohmic device.

The effect of temperature on the $I-V$ characteristics for samples no. 2 and no. 3 is shown in Figures 6 and 7.

In composite varistors, at low temperatures, the conductivity is due to electron transition from donor levels into the conduction band. So the slope of $\ln \sigma \sim 1 / T$ curve in this region is a criterion for measuring the activation energy of donor levels. When the donor levels are depleted from electrons, the resistance of sample increases and its conductance decreases. The activation energy of donor levels for the samples no. 3 and no.4 is $1.27 \times 10^{-2} \mathrm{eV}$ and $1.078 \times 10^{-2} \mathrm{eV}$, respectively, (Figures 8 and 9). That is the distances between the donor level-created by the addition of polymer-and the minimum of conduction band for those samples are $1.27 \times 10^{-2} \mathrm{eV}$ and $1.078 \times 10^{-2} \mathrm{eV}$, respectively.

The higher the polymer contents in the varistor structure, the larger the hysteresis loop. The existence of "dipole moments" in the varistor structure can describe this behavior, (Figures 10 and 11). The hysteresis loop is expanded by the increase of temperature. In the absence of an external electric field, dipole moments of atoms are randomly oriented; they can even be antiparallel. By increasing the electric field, the majority of dipole moments will be oriented along the applied electric field. By decreasing the applied field, apparently a limited number of dipole moments will be oriented along the field direction but most of them however will not be aligned with the field. Therefore, a hysteresis on the $I-V$ characteristics of varistor will be observed.

Expansion of hysteresis loops with the increase in temperature is apparently due to the fact that, under the action of an electric field, there is an orientation of dipole moments along the field; on the other hand, under the action of temperature, an off-orientation of dipole moments will occur, and the probability of their random distribution will increase. This is confirmed by the experimental data through the expansion of hysteresis loop.

Comparison of Figures 10 and 11 also shows that at a constant temperature, with increasing the quantity of polymer in the varistor structure, the hysteresis becomes more expanded. This is due to the increase in the number of dipole moments by increasing the polymer content.

\section{Conclusions}

As long as the nonlinear behavior of the varistor is preserved, the polymer content may be increased in the varistor structure. For the composite varistors with nonlinear $I-V$ characteristic, the increase of threshold voltage depends on the polymer content in the varistor structure.

The resistance of these varistors decreases with increasing the temperature.
The higher the polymer content in the varistor structure, the higher the activation energy and thus the larger the hysteresis loop.

\section{References}

[1] H. Bidadi, et al., in Proceedings of the 5th International Conference on Composite Science and Technology (ICCST '05), pp. 407-412, American University of Sharjah, Sharjah, United Arab Emirates, February 2005.

[2] F. L. Souza, J. W. Gomes, P. R. Bueno, et al., "Effect of the addition of $\mathrm{ZnO}$ seeds on the electrical properties of $\mathrm{ZnO}$-based varistors," Materials Chemistry and Physics, vol. 80, no. 2, pp. 512-516, 2003.

[3] T. K. Gupta, "Application of zinc oxide varistors," Journal of the American Ceramic Society, vol. 73, no. 7, pp. 1817-1840, 1990.

[4] W. G. Carlson and T. K. Gupta, "Improved varistor nonlinearity via donor impurity doping," Journal of Applied Physics, vol. 53, no. 8 , pp. 5746-5753, 1982.

[5] D. R. Clarke, "Varistor ceramics," Journal of the American Ceramic Society, vol. 82, no. 3, pp. 485-502, 1999.

[6] K. Eda, "Zinc oxide varistors," IEEE Electrical Insulation Magazine, vol. 5, no. 6, pp. 28-32, 1989.

[7] http://en.wikipedia.org/wiki/Composite_material.

[8] M. C. Petty, M. R. Bryce, and D. Bloor, An Introduction to Molecular Electronics, Edward Arnold, London, UK, 1955. 

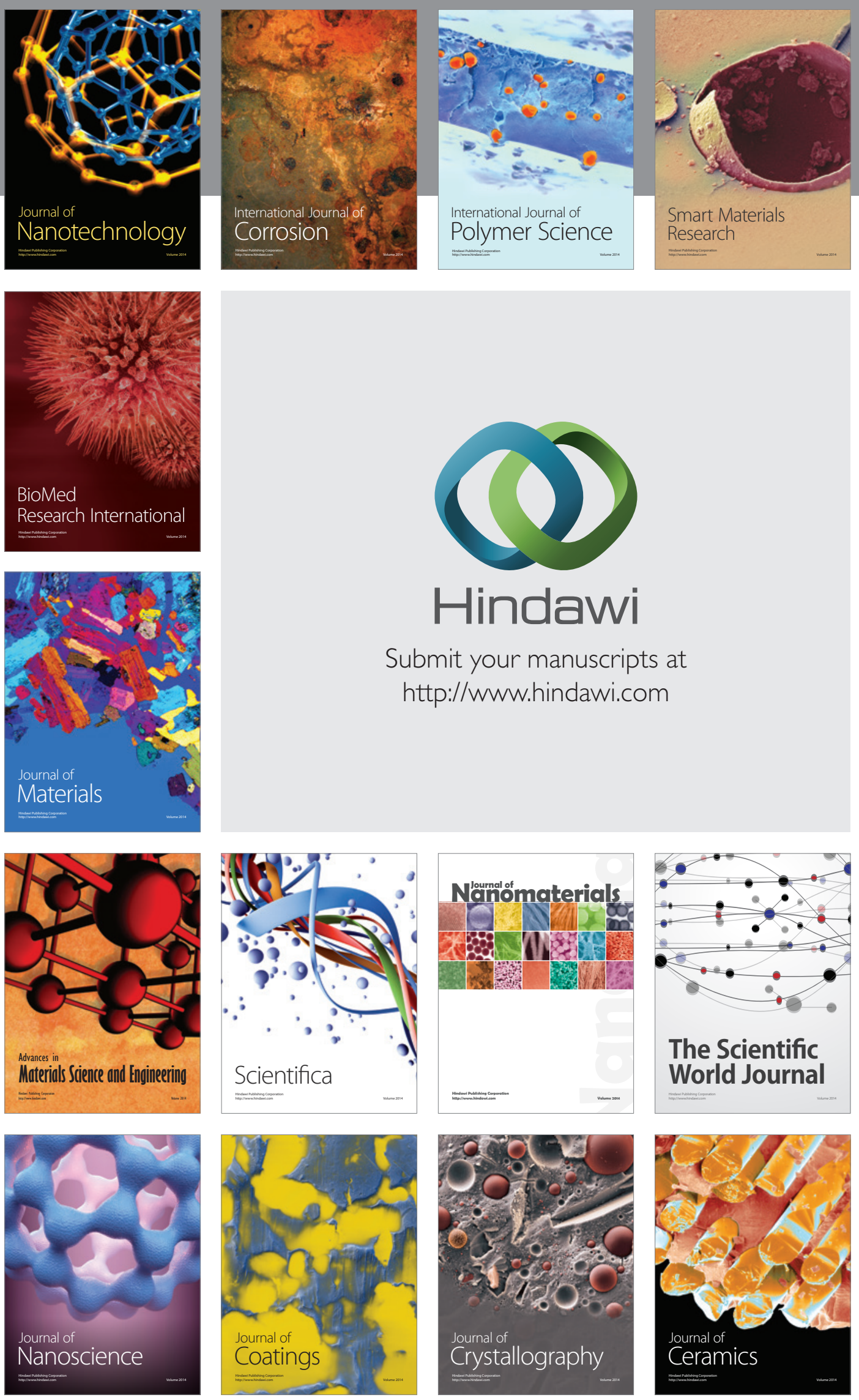

The Scientific World Journal

Submit your manuscripts at

http://www.hindawi.com

\section{World Journal}

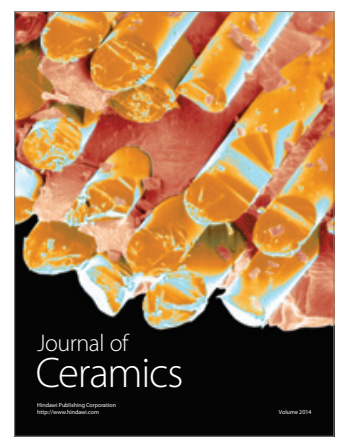

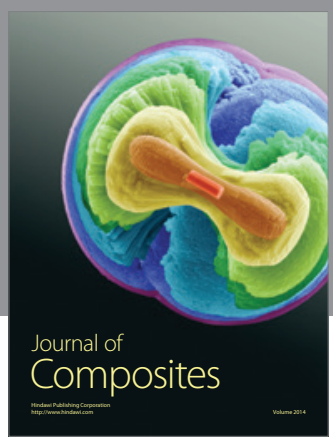
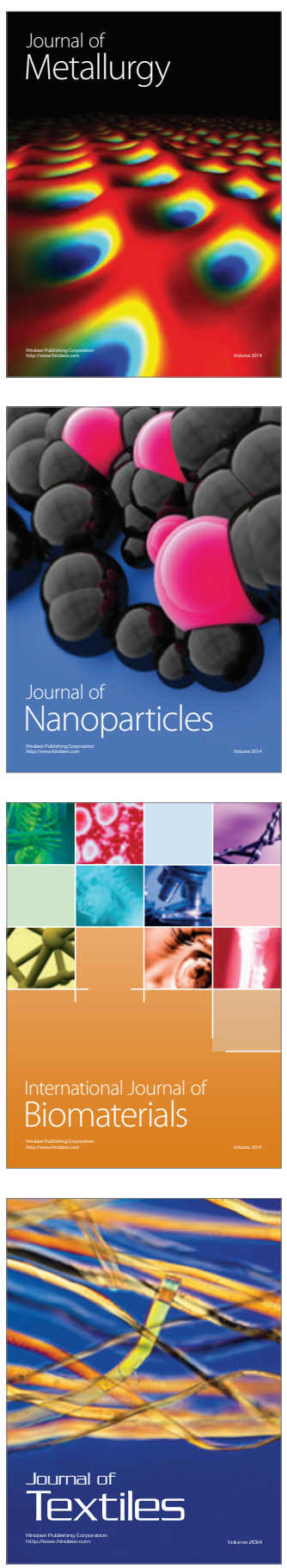